\title{
Identifying Smart Solutions for Fighting Illegal Logging and Timber Trade
}

\author{
Ioannis N. Athanasiadis ${ }^{1,2}$, Despina Anastasiadou ${ }^{2}$, \\ Kostas Koulinas ${ }^{2}$, and Fotis Kiourtsis ${ }^{3}$ \\ 1 Democritus University of Thrace, Xanthi, Greece \\ ioannis@athanasiadis.info \\ 2 Information Technologies Institute, Centre for Research and Technology Hellas, \\ Thessaloniki, Greece \\ ${ }^{3}$ General Directorate of Forests \& Rural Affairs, Decentralized Administration of \\ Macedonia and Thrace, Thessaloniki, Greece
}

\begin{abstract}
We investigate how smart Information and Communication Technologies (ICT) solutions can be used for combating illegal logging and timber trade. We put together techniques from agile requirements engineering to propose a methodology for identifying user stories and associated risks and priorities and via a collaborative, participatory, single day workshop, named inception workshop. We present our findings from the first application of the method, with the active involvement of the relevant stakeholders, i.e technical and domain experts, which concluded in seven user stories.
\end{abstract}

Keywords: Agile methods, requirements engineering, forestry, illegal logging, illegal timber trade, user stories, participatory methods, inception workshop, smart solutions, RFID, low cost sensors.

\section{Introduction}

Illegal logging and timber trade is currently a major concern among all countries world wide [1], while it is acknowledged as a complex problem that involves not only environmental dimension, but also economical and societal ones. While until very recently illegal logging was not an important issue in Greece [2], nowadays it has been intensified due to the economic crisis [3, 4]. In this paper, we investigate how smart Information and Communication Technologies (ICT) solutions can be used for combating illegal logging and timber trade. Following an analysis of the whole logging and timber trade lifecycle, performed with participatory methods and agile techniques, we have identified the weak links, which could potentially be improved by technical solutions.

With the active involvement of the relevant stakeholders, we identified seven user stories for strengthening modus operandi, each one of which introduces one or more ICT technologies. These include electronic documents; barcode and RFID (Radio-frequency identification) tagging; low-cost, low-power sensor networks for motion and volume detection; remote sensing and satellite imaging;

J. Hřebíček et al. (Eds.): ISESS 2013, IFIP AICT 413, pp. 143-153, 2013.

(C) IFIP International Federation for Information Processing 2013 
integrated service-oriented systems. We also identified associated risks and priorities for the implementation pilot of these user stories.

The rest of this paper is organized as follows: Section 2 presents methods and tools, i.e. the problem at hand related to illegal logging and timber trade (par.2.1), agile methods for requirements engineering (par.2.2), and our methodology for identifying and prioritizing solutions (par. 2.3). Section 3 demonstrates the methodology application and our key findings, while section 4 presents with a discussion of our experiences with the process.

\section{Methods and Tools}

\subsection{The Problem at Hand: AITOLOS Project}

The AITOLOS project aims to foster cross-border collaboration and coordinate the activities of Greece and the former Yugoslav Republic of Macedonia against illegal logging and timber trade. Specifically, AITOLOS contributions are towards the following directions:

(a) Assess current legal framework, and define specific recommendations for enhancing legal and regulatory framework in order to fully address legal aspects of illegal logging/trade.

(b) Develop policy guidelines for addressing illegal logging, with a special focus on cross-border timber trade.

(c) Develop an operational plan across participating border regions for implementing policy/guidelines, including the technical specifications, stakeholders roles, monitoring mechanism, timetable and budget.

(d) Engage in communication and dissemination activities at regional,national and European level, presenting developed policy guidelines and regional operational plan.

(e) Two implementation pilots, one per country, in order to assess project results in real world situations. Pilots will run in forest offices of Goumenissa and Bitola.

The project duration is 15 months (2012-2013), and its consortium comprise of the Decentralized Administration of Macedonia \& Thrace, Public Enterprise 'Macedonian Forests', Centre for Research and Technology Hellas (CERTH), Technological Education Institute of Serres (TEIS), and National Association of Private Forest Owners.

One of the major challenges of AITOLOS project was to identify smart ICT solutions to be incorporated into the everyday work of national forest protection services, to facilitate and support traditional processes of forest management, and to protect forest ecosystems against illegal logging and trade; proving that modern technologies can be successfully incorporated into everyday forestry service duties. Identification, prioritization, and risk estimation for building a potential solutions portofolio was a major challenge for the AITOLOS project. Also, selecting which of the potential solutions can be demonstrated as the project pilot was further constrained by our limited amount of resources. To tackle these 
challenges, a bidirectional knowledge shift between forest and computer scientists was required. To develop a common language and achieve consensus among technical and domain scientist, we adopted agile methods originating in software engineering, and put them in action in a single-day, intensive, 'inception' workshop.

\subsection{Agile Methods for Identifying Key Requirements}

Capturing user requirements consist a moving target in projects that are illdefined, or fast-changing, thus the past years agile approaches as Extreme Programming or Scrum have been proposed for capturing requirements informally, i.e. as users stories.

Agile methods for requirements engineering are based on four principles [5]: a) put customers in the center of attention, i.e. involve actively stakeholders through out the process; $b$ ) employ early verification and validation techniques, i.e by developing acceptance tests for user stories, or use cases; $c$ ) identify early non-functional requirements and analyze them with customers; $d$ ) manage change and incorporate it in the process of requirements engineering.

Cao and Ramesh, in [6], presented an empirical study that evaluates agile requirements practices in 16 organizations, and reveals that intensive communication between the developers and customers as the most important one.

Inspired by agile requirements practices, we developed a methodology for engaging end-users early in the process, and applied it in AITOLOS project technical solution identification.

\subsection{The Inception Workshop Approach}

An inception workshop aims to help project participants (i.e. both technology and domain experts) to identify key requirements of the project technical solution. This can be achieved through eliciting initial user stories, which will be subsequently developed through iterations. Domain and technology experts interact in a single day intensive workshop, in order to come to a common understanding as to the scope of the project technical solution.

Participant roles participants are domain and technology experts, and a facilitator. Domain experts participate in order to present their application domain and present problems in their everyday work practice. Technology experts come to the workshop for understanding better the problem domain and specify the technical solution The facilitator is responsible for running the workshop according to schedule and keep discussions focused.

The inception workshop is organized in three sessions. Figure 2.3 presents a typical agenda of an inception workshop.

The first session focuses on domain understanding, in our case forestry and illegal logging. The goal of this session is to detail the current processes followed, and increase the understanding of the technical experts. Domain experts share their knowledge in a story-telling fashion: They communicate their knowledge 


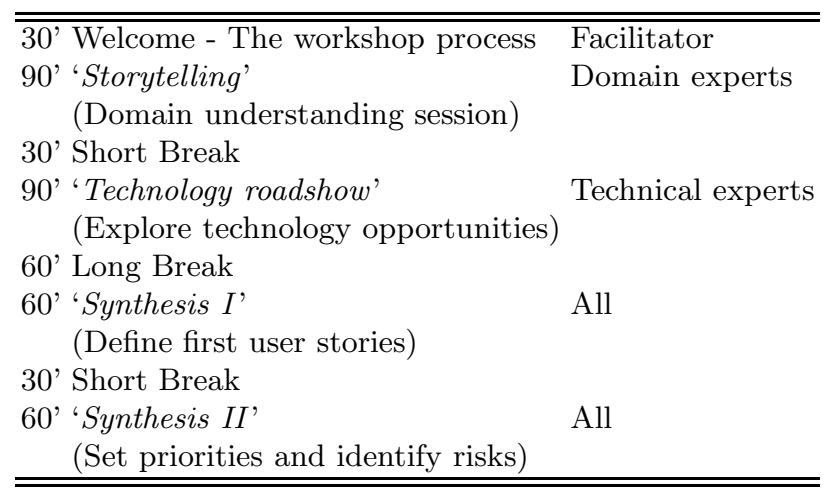

Fig. 1. Typical agenda of an inception workshop (activities with durations)

by sharing experiences and anecdotes. In a collaborative team environment, domain experts stand up to tell the story of their every day work, in a procedural manner, while technology experts intervene with clarifying questions. The facilitator needs to keep the storyline progressing, and prevent the domain experts from diving into details.

The second session focus is on understanding technology opportunities and identifying technology solutions. Through a brainstorming process, technical experts 'put guns on the table', and exemplify how technical solutions might be used for fighting illegal logging. Existing or new solutions are presented informally by technical experts, in order to increase domain experts awareness of the capabilities of ICT systems, and their application in the domain. In a technology roadshow, technical experts present solutions in the form of examples, not only related to the domain at hand, but from other domains as well. Domain experts are free to ask questions, so that they understand what technology tools may offer, and the facilitator is responsible for moderating jargon language and reminding technical experts to use simple examples from known applications to the domain experts.

The final, third, session aim is to produce a synthesis of the two views, the one of the domain experts with that of technology experts. In a collaborative team environment, domain and technology experts identify together initial user stories. Agile requirements methods consider user stories as very high-level definition of requirements, containing just enough information so that the developers can produce a reasonable estimate of the effort to implement it [7]. In our case, initial user stories aim to produce very high-level requirements of technical solutions for the project at hand. While identifying those, we also set priorities in terms of effectiveness, risk and cost. 


\section{Demonstration: The AITOLOS Inception Workshop}

For identifying the potential technical solutions of the AITOLOS project (see par. 2.1), we organized the first AITOLOS inception workshop, in Thessaloniki, Greece, on January 25, 2013, with the participation of all Greek project partners. Approximately 12 domain experts from two forest services (Kilkis and Goumenissa), and 6 technology experts from CERTH and TEIS participated with the main goal of answering the question: 'How smart ICT solutions may be applied in forest service practice in order to combat illegal logging and timber trade?'. We had a very vibrant and interesting discussions in the form of an 'inception workshop'. In the following paragraphs, we present our findings from the workshop. Storytelling and Technology roadshow sessions are presented with annotations from bibliography, to strengthen the opinions that have been revealed during the workshop. Synthesis session presents our results in terms of the technical solutions identified, presented here as user stories, annotated with our estimates in terms of risks and costs.

\subsection{Storytelling}

During the storytelling session, forest scientists presented the whole lifecycle of wood, from the forest to the timber market. Illegal logging activities include the harvest, transportation, purchase or sale of timber in violation of national laws. The harvesting procedure itself may be illegal, including using corrupt means to gain access to forests; extraction without permission or from a protected area; the cutting of protected species; or the extraction of timber in excess of agreed limits [8].

We went through five steps in the timber logging and trade value chain: a) Timber pre-marking, b) Felling and pilling, c) Delivery and transport, $d$ ) Storage (and trade) e) Cross-border timber trade. We highlighted differences in the process between fuel wood and technical wood. Our findings are summarized in Figure 2,

Issues related to illegal logging were presented, and verified that 'two types of illegal logging can be clearly distinguished in South-East Europe countries: (a) poverty driven; and (b) commercial. These types of illegal logging generally take place in different forest areas, are carried out by different offenders, and have different impacts. For example, poverty-driven illegal logging is typically carried out in remote locations, out of sight of the state forest management and law enforcement bodies' [9]. Domain experts provided with evidence from their areas and detailed incidents of illegal logging and timber trade of the two types. We concluded that in Greece, poverty-driven illegal logging typically involves fuel-wood, while commercial illegal logging is related to both fuel-wood and technical wood.

At the same time, we realized that Greece is not an exception to the UN report observation that in South-East Europe many countries have weak forest information systems [10]. Most forest services in Greece are largely doing their business with 'pen and paper', and digitization of processes and documents are 


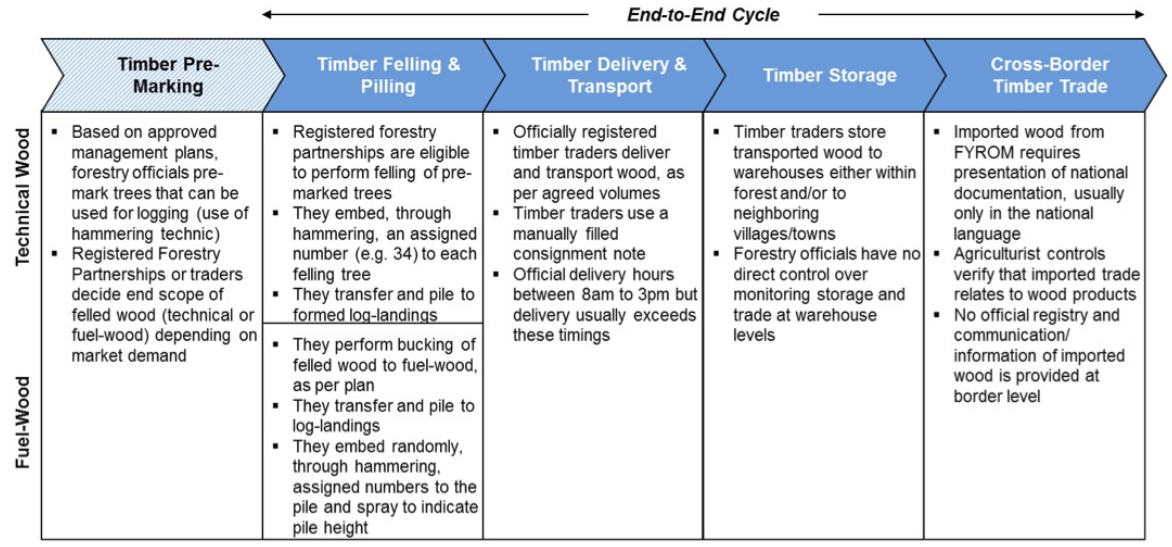

Fig. 2. Workshop notes on timber logging and trade value chain

still in its infancy. Wood value chain in Greece remains undeveloped and several obstacles remain related to official forest maps, forest data recording and cadastral, adaptation of EU specifications, and producing official forest maps, forest data recording, rapid adaptation to EU specifications [11].

\subsection{Technology Roadshow}

During the technology roadshow session, technical scientists with different backgrounds presented relevant technologies that could be potentially used in improving the situation with illegal logging and timber trade. Among those are the followings:

Central systems for issuing permits: A recent Interpol/UNEP report [1] recommends to centralize nationally the issuing of permits for land clearance whether for logging, plantations or ranching, and permits for road transport of timber with strong anti-counterfeit measures.

DNA fingerprinting: The use of ambient sonic spray ionization mass spectrometry as phytochemical markers [12], and DNA fingerprinting [13 17] has been reported for identifying timber origin, and verifying timber supply chains integrity, especially for high-valued timber. Such forestry forensics tools can be proved effective as a means against illegal logging, however the effects of population substructure and inbreeding [18] need to be taken under account.

Market control mechanisms include the expansion of market inspection competencies to ensure the complete control of documentation accompanying shipments of wood, including statements of origin and logging permits [9].

Forest certification: Chain of custody control measures ensures that illegally sourced wood cannot be processed in plants, as some proof of origin certification is required. Forest governance and overall forest preservation can 
be improved through the introduction of certificates for sustainable forest management and chain of custody certificates [9].

GIS systems and databases: Field computers, GPS receivers and GIS databases can ensure the constant monitoring of wood flow and so prevent illegal trade [9].

Marking logs with RFID tags: Pulping compatible, passive UHF transponder made of a wood composite material was developed for marking of logs together with robust RFID readers for the harvester and saw mills [19].

Satellite imagery at multiple temporal and spatial resolutions can effectively increase transparency in the forestry sector by revealing the rate and extent of deforestation on an annual basis and identifying potential areas of illegal logging [20].

Machine vision: Wood identification using anatomical characteristics can be automated through machine vision, as reported in 21], that could be used for fraud detection.

Technology experts presented the above-mentioned technologies, giving examples from forestry applications abroad and from scientific literature, along with examples from other domains with which our domain experts were familiar with. These included market retail, taxation and fiscal systems, electronic tickets, parking stations, and fire protection.

\subsection{Synthesis: User Stories, Risks, Priorities}

The previous two sessions increased confidence of our technology experts with illegal logging and timber trade issues, and our domain scientists with modern ICT technologies, and their potential in forest protection. Following a longer break were informal discussions continued, we reconvened in collaborative session where we started shaping solution ideas into the following user stories. The order of presentation is not significant.

S1: Electronic documents with security characteristics. All documents involved in timber transportation (from the log landing areas to the market) becomes digital, to avoid fraudulent documents. This involves both logging permits and timber transportation notes (which accompany trucks from log landing areas to timber warehouses). New document templates incorporate security features in the form of barcode, QR code, or e-signatures, which are instantly verifiable. Using low cost smart devices, security features can be verified online, via accessing web addresses over the web.

Risk assessment and priority: This solution is easy to implement and resembles an electronic ticketing mechanism with several control points. Legal enforcement and information security infrastructure are the main challenges of this solution.

S2: Smart tags for timber marking. Currently, timber marking is performed with hammers that mark wood with forest office, or forester code. This is a weak point that can be significantly improved by introducing unique tags 
for all wood marked by hammer. This can be achieved by using plastic barcode or RFID tags, or RFID nails from pulping compatible material. This solution is ideal both for proving technical wood log origin, and for timber certification.

Risk assessment and priority: RFID technology has been extensively applied in market applications, however there is little experience with timber marking. In this respect, reading capacity of equipment needs to be verified in the environmental conditions of log landing areas. At the same time, there are operational challenges related to who applied the tags, who bears the cost of the application and how to check tags when logs are loaded on trucks. Last but not least, information security and cryptographic characteristics of the information encoded in the tags needs to be studied in depth.

S3: Cross-border uniform, multilingual reports. Forms attached to timber imported (or exported) are standardized, and all texts appear in both local languages, and English. This solution removes language barriers at inspection points and could be achieved by developing a common standardized template to be shared between the neighboring countries.

Risk assessment and priority: While seems simple, due to the different policies and regulations of the two countries, achieving consensus might be a problem. Enforcement and orchestrating all involved authorities in both countries could be troublesome too. Also, when exported in processed form, timber becomes a marketable product. This implies that European common market rules apply, and forest regulations do not apply any longer, rather is a matter of custom inspection and international trade.

S4: Truck load detection. Manual estimation of timber volume loaded on trucks is another weak point of the value chain. Using an one-stop gate, equipped with low cost, low power sensors to calculate automatically wood volume loaded on a truck. This could be done for example by two depth cameras getting two viewpoints of a truck: top and rear, and detecting the contour of the volume loaded.

Risk assessment and priority: While today there are several cheap sensors and this solution seems easy to deploy, our estimation was that it is gonna be hard to calibrate with accuracy, and truck type knowledge might be needed. To the best of our knowledge there are no similar applications, at least reported in literature, so a prototype is needed to evaluate the proof of concept.

S5: Truck motion detection. While effective monitoring of timber transport and access control to forest areas have been identified as a key to combat illegal logging, this is a very difficult task, as forests are not closed and a dense network of dirt roads and paths exist. However, as a forest service we would like to see implemented a typical fleet management scenario, that uses GPS/GSM network to detect truck routes, and report them online. Similar approaches might use RFID tags or automatic vehicle identification (AVI) at forest gates to patrol entrance/exit from certain checkpoints.

Risk assessment and priority: While this solution could be extremely effective, transporters consent would be required and privacy issues may arise. 
Also implementation in forest conditions are not well studied, and poor signal coverage may affect efficiency.

S6: Satellite imaging. This story considers the application of image processing algorithms on satellite images to detect areas of intervention and protection. Such tools have been applied extensively in forest fire monitoring. In our case, we identified two scenarios. The operational scenario, where we use images on demand, in order to identify illegal logging at the time it happens, and the strategic scenario, where the same techniques may apply on low-cost, historical images, to estimate forest degradation due to illegal logging and prioritize future intervention actions.

Risk assessment and priority: We need to keep a balance between image cost and resolution needed. A high-resolution, on demand image cost is extremely expensive, thus the operational scenario seem not viable, at least for fuel wood related illegal activities.

S7: Systems integration. In the current situation, information doesn't flow across the borders, neither between the various national services involved in the inspection of timber trade value chain (i.e customs, taxation, police and forest authorities). As a forest service we need an online system that links customs with forest services, and can notify forest services on vehicle movements across borders, and report on volume and type of wood, origin and destination, etc.

Risk assessment and priority: While this seems from a technical point of view a relatively straightforward solution, we identify risks related to data content, system interoperability and standardization, and the implementation of an appropriate information security infrastructure.

\section{Discussion}

The inception workshop helped a lot our project members to develop a common language and understand better each other expertise. It was also proven helpful in building team spirit due to the participatory sessions, where interactions brought our team closer.

We also managed to identify early in the project, what is more important to our own users, rather than considering generic literature cases or an average hypothetical user.

Additionally, before and after the workshop we conducted a survey to evaluate participant perceptions. Currently we process the survey results, and in general we identified that there was a knowledge shift in both domain and technical scientists. Also, participant feedback on the process was very positive.

We identified that a main issue during our AITOLOS inception workshop was the large number of participants. This made discussions last longer than expected, but on the other hand, it was an important factor in producing seven user stories in a single day. Future plans include organizing a second inception workshop with rest of the project partners, and we intend to repeat the same process with half of the participants, reducing the total number of participants to ten (i.e 3-4 technical experts, 5-6 domain experts, 1 facilitator). 
Last, but not least, the user stories we identified with the inception workshop are epic, in the sense that they can be broken down to smaller, more targeted stories. However, this was rather expected as the goal of the inception workshop was not to identify certain features to be implemented, rather it was to identify systems to be subsequently specified in detail.

Acknowledgments. Authors would like to thank the personnel of the Forest Service Offices of Goumenissa and Kilkis, and the technical experts from Centre for Research and Technology Hellas, and Technological Education Institute of Serres for their participation to the inception workshop.

This work have been partially supported by the IPA cross-border programme 'Greece-The former Yugoslav Republic of Macedonia 2007-2013' under grant AITOLOS, which is co-funded by the European Union and by national funds of the participating countries.

More about the project at http://www.aitolos.eu.

\section{References}

1. Nellemann, C.: INTERPOL Environmental Crime Programme. In: Carbon, G. (ed.) Black Trade: Illegal Logging, Tax Fraud and Laundering in the Worlds Tropical Forests. A Rapid Response Assessment. United Nations Environment Programme, GRID-Arendal (2012), www.grida.no

2. Ministry of Rural Development and Food: National report of Greece concerning the illegal logging and the trade of the illegally produced forest products. Illegal logging and trade of illegally-derived wood products in the UNECE region: Causes and extent, UNECE \& FAO (2004), http://www.unece.org/fileadmin/DAM/ timber/docs/sem/2004-1/full_reports/Greece.pdf

3. Papachristou, H.: Greek austerity fuels illegal logging, ministry says. News article, Reuters (2012), http://www.reuters.com/article/2012/09/18/greecelogging-idUSL5E8KIKAY20120918 (Published online on) (September 18, 2012)

4. Lowen, M.: Greece crisis: Poor turn to illegal logging to survive. News Article, BBC (2013), http://www.bbc.co.uk/news/world-europe-21227572 (Published online on January 28, 2013)

5. Eberlein, A., Leite, J.: Agile requirements definition: A view from requirements engineering. In: Proceedings of the International Workshop on Time-Constrained Requirements Engineering (TCRE 2002), pp. 4-8 (2002)

6. Cao, L., Ramesh, B.: Agile requirements engineering practices: An empirical study. Software, IEEE 25, 60-67 (2008)

7. Cobb, C.G.: Making Sense of Agile Project Management. John Wiley and Sons (2011)

8. Marijnissen, C., Ozinga, S., Richards, B., Risso, S.: Facing Reality: How to halt the import of illegal timber in the EU. Technical report, FERN - Greenpeace European Unit - WWF European Policy Office (2004)

9. Markus-Johansson, M., Mesquita, B., Nemeth, A., Dimovski, M., Monnier, C., Kiss-Parciu, P.: Illegal Logging in South Eastern Europe. REC Working Paper, Regional Environmental Center for Central and Eastern Europe (2010)

10. UN: The European Forest Sector Outlook Study II 2010-2030. Technical report, UNECE, FAO (2011) 
11. Koulelis, P.P.: Greek timber industries and wood product markets over the last century: Development constraints and future directions. Annals of Forest Research 54, 229-240 (2011)

12. Cabral, E., Simas, R., Santos, V., Queiroga, C., Da Cunha, V., De Sa, G., Daroda, R., Eberlin, M.: Wood typification by Venturi easy ambient sonic spray ionization mass spectrometry: The case of the endangered Mahogany trees. Journal of Mass Spectrometry 47, 1-6 (2012)

13. Lowe, A., Wong, K.N., Tiong, Y.S., Iyerh, S., Chew, F.T.: A DNA method to verify the integrity of timber supply chains; Confirming the legal sourcing of merbau timber from logging concession to sawmill. Silvae Genetica 59, 263-268 (2010)

14. Nowakowska, J.: Application of DNA markers against illegal logging as a new tool for the Forest Guard Service. Folia Forestalia Polonica, Series A 53, 142-149 (2011)

15. Jolivet, C., Degen, B.: Use of DNA fingerprints to control the origin of sapelli timber (Entandrophragma cylindricum) at the forest concession level in Cameroon. Forensic Science International: Genetics 6, 487-493 (2012)

16. Degen, B., Ward, S., Lemes, M., Navarro, C., Cavers, S., Sebbenn, A.: Verifying the geographic origin of mahogany (Swietenia macrophylla King) with DNAfingerprints. Forensic Science International: Genetics 7, 55-62 (2013)

17. Ganopoulos, I., Aravanopoulos, F., Madesis, P., Pasentsis, K., Bosmali, I., Ouzounis, C., Tsaftaris, A.: Taxonomic Identification of Mediterranean Pines and Their Hybrids Based on the High Resolution Melting (HRM) and trnL Approaches: From Cytoplasmic Inheritance to Timber Tracing. PLoS ONE 8 e60945 (2013)

18. Tnah, L., Lee, S., Ng, K., Faridah, Q.Z., Faridah-Hanum, I.: Forensic DNA profiling of tropical timber species in Peninsular Malaysia. Forest Ecology and Management 259, 1436-1446 (2010)

19. Häkli, J., Jaakkola, K., Pursula, P., Huusko, M., Nummila, K.: UHF RFID based tracking of logs in the forest industry. In: RFID 2010: International IEEE Conference on RFID, pp. 245-251 (2010)

20. Fuller, D.: Tropical forest monitoring and remote sensing: A new era of transparency in forest governance? Singapore Journal of Tropical Geography 27, 15-29 (2006)

21. Hermanson, J., Wiedenhoeft, A.: A brief review of machine vision in the context of automated wood identification systems. IAWA Journal 32, 233-250 (2011) 